\title{
Article \\ A Metabolic Profiling Analysis Revealed a Primary Metabolism Reprogramming in Arabidopsis glyI4 Loss-of-Function Mutant
}

\author{
Silvia Proietti ${ }^{1,+}\left(\mathbb{D}\right.$, Laura Bertini $^{1,+}{ }^{,}$, Gaia Salvatore Falconieri ${ }^{1}\left(\mathbb{D}\right.$, Ivan Baccelli ${ }^{2}\left(\mathbb{D}\right.$, Anna Maria Timperio ${ }^{1, *(\mathbb{D})}$ \\ and Carla Caruso ${ }^{1, *(1)}$ \\ 1 Department of Ecological and Biological Sciences, University of Tuscia, 01100 Viterbo, Italy; \\ s.proietti@unitus.it (S.P.); lbertini@unitus.it (L.B.); gfalconieri@unitus.it (G.S.F.) \\ 2 Institute for Sustainable Plant Protection, National Research Council of Italy, Sesto Fiorentino, 50019 Florence, \\ Italy; ivan.baccelli@ipsp.cnr.it \\ * Correspondence: timperio@unitus.it (A.M.T.); caruso@unitus.it (C.C.); Tel.: +39-0761-357330 (C.C.) \\ + These authors contributed equally to this work.
}

Citation: Proietti, S.; Bertini, L.; Falconieri, G.S.; Baccelli, I.; Timperio, A.M.; Caruso, C. A Metabolic Profiling Analysis Revealed a Primary Metabolism Reprogramming in Arabidopsis glyI4 Loss-of-Function Mutant. Plants 2021, 10, 2464. https://doi.org/10.3390/ plants10112464

Academic Editors: Daniel-Valentin Savatin, Francesco Sestili and Thomas Nägele

Received: 28 September 2021 Accepted: 11 November 2021 Published: 15 November 2021

Publisher's Note: MDPI stays neutral with regard to jurisdictional claims in published maps and institutional affiliations.

Copyright: (c) 2021 by the authors. Licensee MDPI, Basel, Switzerland. This article is an open access article distributed under the terms and conditions of the Creative Commons Attribution (CC BY) license (https:// creativecommons.org/licenses/by/ $4.0 /)$.

\begin{abstract}
Methylglyoxal (MG) is a cytotoxic compound often produced as a side product of metabolic processes such as glycolysis, lipid peroxidation, and photosynthesis. MG is mainly scavenged by the glyoxalase system, a two-step pathway, in which the coordinate activity of GLYI and GLYII transforms it into D-lactate, releasing GSH. In Arabidopsis thaliana, a member of the GLYI family named GLYI has been recently characterized. In glyI4 mutant plants, a general stress phenotype characterized by compromised MG scavenging, accumulation of reactive oxygen species (ROS), stomatal closure, and reduced fitness was observed. In order to shed some light on the impact of gly4 loss-of-function on plant metabolism, we applied a high resolution mass spectrometry-based metabolomic approach to Arabidopsis Col-8 wild type and glyI4 mutant plants. A compound library containing a total of 70 metabolites, differentially synthesized in glyI4 compared to Col-8, was obtained. Pathway analysis of the identified compounds showed that the upregulated pathways are mainly involved in redox reactions and cellular energy maintenance, and those downregulated in plant defense and growth. These results improved our understanding of the impacts of glyI4 loss-of-function on the general reprogramming of the plant's metabolic landscape as a strategy for surviving under adverse physiological conditions.
\end{abstract}

Keywords: methylglyoxal; glyoxalase I; metabolite profiling; oxidative stress; plant growth; plant defense

\section{Introduction}

During evolution, plants have evolved sophisticated mechanisms allowing adaptation and survival under biotic and abiotic stress conditions [1,2]. After sensing the stimulus, plants activate general reprogramming of the genetic machinery and of the metabolic profile, leading to efficient defense responses and ultimately to plant tolerance [2]. The finely tuned defense mechanisms are substantially orchestrated by small molecules, such as phytohormones, plant volatile organic compounds (VOCs), and primary and secondary metabolites [3-5]. Besides, plants exposed to stresses often produce toxic compounds, such as methylglyoxal (MG) — one of the most ubiquitous toxins produced [6-10]. MG is a reactive alpha-ketoaldehyde formed as a side product of metabolic processes such as glycolysis, lipid peroxidation, photosynthesis, and protein degradation [11]. In particular, a high concentration of MG affects seed germination, plant growth and development, and photosynthesis [11,12]. It can also regulate stomatal closure by triggering accumulation of reactive oxygen species (ROS) in the guard cells [13]. Noteworthy, MG spontaneously glycates nucleic acids, proteins, and lipids, forming advanced glycation end products (AGEs) that are involved in oxidative stress onset and can alter the expression of signal transduction-related genes involved in cellular metabolism and transport, protein degradation, and stress/defense responses [11]. To limit the damage caused by MG, an important 
detoxification mechanism ensured by the glyoxalase (GLY) system has evolved. In particular, the MG detoxification process is mostly mediated by two enzymes: glyoxalase I (GLYI) and glyoxalase II (GLYII). GLYI leads to the formation of S-lactoylglutathione, which is subsequently transformed into D-lactate by GLYII, regenerating GSH in the process [13]. By facilitating MG detoxification, plant glyoxalases can be conveniently assigned to diverse physiological roles, which are involved in the detox central pathway for sugar metabolism and other primary physiological processes [12]. In plant species such as rice, tobacco, and tomato the overexpression of glyoxalase genes, either individually (GLYI or GLYII) or as a complete pathway $(G L Y I+G L Y I I)$, lead to plants with improved tolerance of biotic, abiotic, and oxidative stresses [12,14-16]. Several genes belonging to the GLYI and GLYII families have been isolated and characterized in many plants. For instance, 11 GLYI genes, members of the vicinal oxygen chelate (VOC) superfamily, were characterized in Arabidopsis [17]. Recently, we thoroughly studied AtGLYI4, a member of the Arabidopsis GLYI family. A genome-wide association (GWA) study suggested glyoxalase AtGLYI4 as a novel player in defense hormone signaling and in defense against biotic stresses in Arabidopsis $[18,19]$. Moreover, it has been shown that AtGLYI4 has a great impact on MG scavenging and on plant health. In fact, in the T-DNA insertion line glyI4, compromised MG scavenging, ROS accumulation, stomatal closure, and reduced plant fitness were observed [19]. Additionally, accumulation of MG led to lower efficiency of the jasmonate signaling pathway and increased the susceptibility of glyI4 plants to the necrotrophic fungus Plectospherella cucumerina [19].

Under adverse conditions, general reprogramming of signaling and defense responses is widely recognized [20]. Moreover, it is reasonable that plants execute metabolic rewiring too, in order to restore chemical and energetic balances essential for acclimation and survival. Therefore, metabolic profiling analysis can be a valid approach through which to accurately understand the different processes that take place in the plant during stressful conditions. Indeed, metabolites are the results of both biological and environmental factors, and provide great potential to bridge our knowledge about genotypes and phenotypes. Many studies revealed that metabolic activities respond to stress faster than transcriptional activities, thereby making metabolic changes an important component of early stress responses [21]. Moreover, metabolomic approaches are useful to discover novel genes or new gene functions and to characterize the metabolic responses to stress conditions [20]. Finally, the idea that untargeted mass spectrometry (MS)-based metabolomic analysis will result in a large list of identified small molecules that can be mapped to networks and pathways is often assumed [22].

In this work, we aimed to investigate the metabolic profiling of a glyI4 loss-of-function mutant against Col- 8 wild type to unravel the blend of metabolites and the pathways they are involved in, which could further explain the impacts of glyI4 loss-of-function on the altered physiological processes that we previously observed. Results of LC-MS/MS analysis showed differentially expressed metabolites in glyI4 mutant with respect to Col-8. Pathway enrichment analysis revealed that the upregulated pathways are mainly involved in redox reactions and in cellular energy maintenance, while the downregulated ones in plant growth and defense. Furthermore, downregulation of marker genes involved in plant growth and defense, such as PAL2, CAD4, and EPSPS, was observed in the glyI4 mutant. Finally, focusing on redox reactions, enzymatic assays confirmed the impairing of ROS scavenging in the glyI4 mutant. These results corroborated the role of GLYI4 in the trade-off between plant survival and defense.

\section{Results and Discussion}

\subsection{GLYI4 Knock-Down Affects Plant Metabolism}

In the present study, we performed high-throughput metabolic profiling analysis through LC-MS/MS, which allowed us to identify 131 metabolites, among which 70 were differentially synthesized $\left(\log _{2}\right.$-fold change $>|1|$, $p$-value $\left.<0.05\right)$. In detail, 23 of them were over-produced and 47 were under-produced in the glyI 4 mutant compared to Col- 8 
(Supplementary Table S1). The metabolome overview obtained through the Metaboanalyst 5.0 "pathway analysis" tool showed "arginine biosynthesis," "pyrimidine metabolism," and "cysteine and methionine metabolism" pathways as the three most upregulated metabolic pathways in glyI4 compared to Col-8, based on $p$-value $<0.05$ and pathway impact $>0$ (Figure 1).

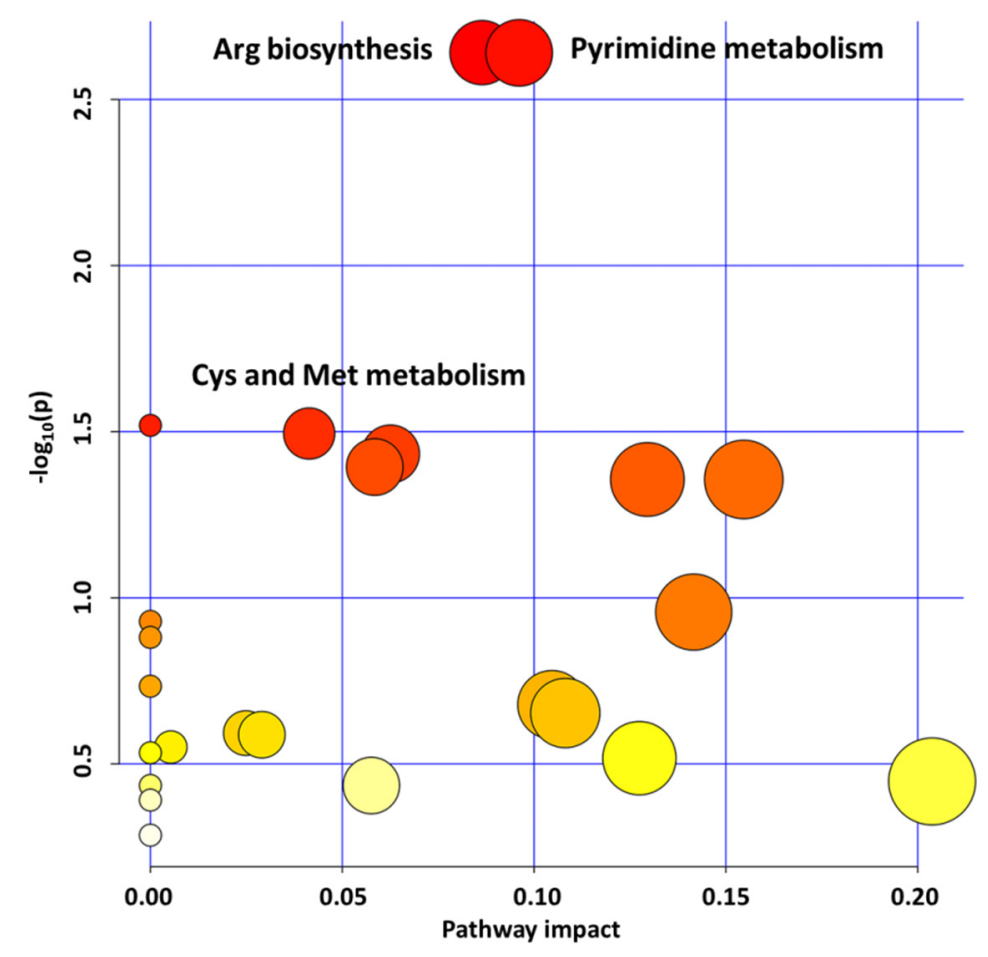

Figure 1. Metabolic pathway analysis plot. Color intensity (white to red) reflects increasing statistical significance, and circle diameter varies with the pathway impact. The graph was made by plotting on the $y$-axis the $-\log _{10}$ transforms of $p$-values from the pathway enrichment analysis and on the $x$-axis the pathway impact values derived from the pathway topology analysis. The top three most significant pathways ( $p$-value $<0.05$; pathway impact $(\mathrm{pi})>0$ ) are depicted: arginine biosynthesis $(p$-value $=0.0022868, \mathrm{pi}=0.08641) ;$ pyrimidine metabolism $(p$-value $=0.0022904, \mathrm{pi}=0.09611) ;$ cysteine and methionine metabolism $(p$-value $=0.032033$, pi $=0.04138)$. The metabolomic analysis was repeated three times using different biological samples giving the same results.

Moreover, among the first top-ten significantly uniquely enriched pathways were "citrate cycle (TCA cycle)," "carbon fixation in photosynthetic organisms," "alanine, aspartate and glutamate metabolism," "pyruvate metabolism," "glycerophospholipid metabolism," and "monobactam biosynthesis".

\subsubsection{Pyrimidine Metabolism Upregulation Influences Redox Homeostasis}

MG has been found to affect pyrimidine metabolism in human cells [23], whereas in plants its effect on nucleotides biological processes is still unknown.

Nucleobases, such as pyrimidines and purines, are important nitrogen compounds constituting the building blocks necessary for DNA and RNA synthesis; energy sources; coenzymes for redox reactions; and precursors for the synthesis of primary and secondary products, such as isobarbituric acid, glycosides, and $\beta$-alanine [24]. Furthermore, pyrimidines play a key role as regulators of amino acid, phospholipid, glycolipid, sugar, and polysaccharide biosynthesis [25]. Pyrimidines are synthetized from amino acids, and small molecules through de novo pathways and from preformed nucleobases and nucleosides through salvage pathways [26]. Since they have important functions in a multitude of biochemical and developmental processes, they are essential during the entire life cycle of the plant. Among all metabolites of pyrimidine metabolism pathway, our attention 
has been focused on the dihydroorotic acid, which is involved in redox reactions. This metabolite appears to be localized at the mitochondrial level, and this is very interesting, since mitochondria are essential regulators of redox-dependent processes [27]. In fact, in this cellular compartment, redox-sensitive processes occur, providing a rapid response to metabolic changes and reactive oxygen species (ROS) fluxes [28]. The over-production of this metabolite in the glyI4 mutant could be linked to the previously observed high ROS production in this mutant [19]. Pyrimidine metabolism is also involved in the $\beta$-alanine biosynthesis, which was another upregulated pathway in the glyI4 mutant, although its impact value was below the significant threshold.

\subsubsection{Aminoacid Metabolism Upregulation Promotes Cellular Energy Maintenance}

It has been proved that MG impacts the metabolism of amino acids in rat cerebral tissues [29]. In our work, we found that among the most significant perturbed pathways are those related to "arginine biosynthesis" and "cysteine and methionine metabolism." In most terrestrial habitats, nitrogen is a limiting resource for plant growth, since large amounts of nitrogen are needed to synthesize proteins and nucleic acids. Among the 21 proteinogenic amino acids, arginine has the highest nitrogen/carbon ratio, which makes it especially suitable as a form of organic nitrogen storage. Biosynthesis in chloroplasts via ornithine is apparently the only pathway to provide arginine in plants, and the rate of arginine synthesis is tightly regulated by various feedback mechanisms in accordance with the overall nutritional status [30]. Although several steps of arginine biosynthesis still remain poorly characterized in plants, its role in maintaining the energy status of the cell is apparent [31]. Cysteine and methionine metabolism plays a significant role in the biosynthesis of different biomolecules, such as glutathione, ethylene, phytochelatines, polyamines, and biotin [32]. In particular, cysteine is crucial for many biosynthetic pathways, and it is a precursor molecule of diverse sulfur-containing metabolites [33]. In general, cysteine is the direct or indirect donor of all reduced sulfur groups in plant cells. In Arabidopsis, the most important ones are methionine, iron-sulfur cluster, glutathione, molybdenum cofactor, vitamins (coenzyme A, lipoic acid, thiamine, biotin), and secondary compounds such as camalexin and glucosinolates [34]. It has been already reported elsewhere that to cope with a stress, glyoxylases are essential to decrease the methylglyoxal level and increase reduced glutathione and cysteine [35]. In our previous work, we observed deprivation of GSH in glyI4 as an effect of impaired methylglyoxal detoxification [19]. Interestingly, in our metabolite dataset, we found cystine (Supplementary Table S1). Taken together, the fact that in our model system we observed a GSH decrease and cystine enrichment in glyI4 could be a sign of the compromised plant health due to MG accumulation. Hence, the results of metabolomic analysis strengthen our previous findings, by using a different approach. Methionine is another important amino acid, belonging to the aspartate family that is involved in plant metabolism, and being part of the protein structure. In Arabidopsis, recent studies have shown that methionine affects the metabolic regulation of other pathways, such as polyamines, ethylene, and glucosinolate pathways [36]. In particular, methionine is synthesized by the aspartate family pathway which leads to the formation of other amino acids, such as threonine and isoleucine. These amino, acids take part in protein synthesis, and additionally, they can be catabolized into the TCA cycle in order to contribute to cellular energy metabolism [37]. Altogether, these results led us to speculate that a general reprogramming towards energy-generating processes could occur in the glyI4 mutant, allowing the plant to have more energy to adapt, survive, and cope with stressful conditions.

2.1.3. Purine, Aromatic Amino Acid, and Zeatin Biosynthesis Downregulation Affects glyI4 Mutant Growth and Defense

We also analyzed the enriched pathways in the under-produced metabolites dataset in the glyI4 mutant compared to Col-8. As shown in Figure 2, we focused our attention on the top three most significant enriched pathways: "purine metabolism," "phenylalanine, tyrosine, tryptophan metabolism," and "zeatin biosynthesis". 


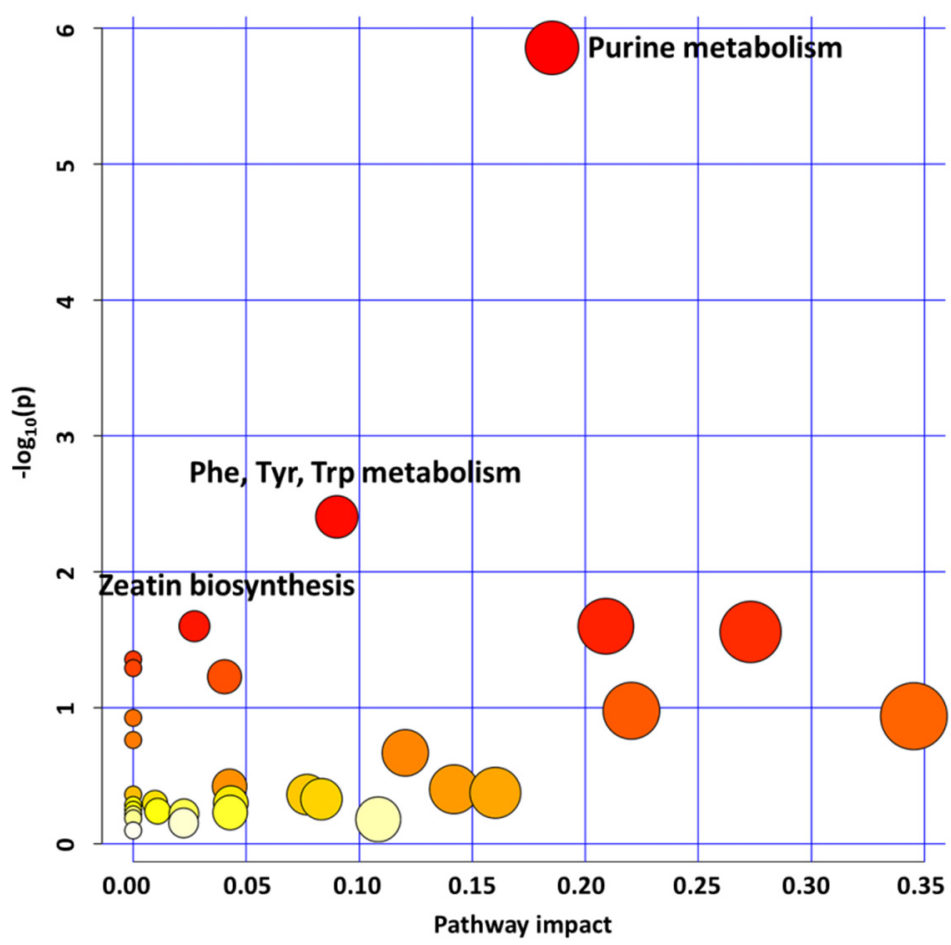

Figure 2. Metabolic pathway analysis plot. Color intensity (white to red) reflects increasing statistical significance, and circle diameter varies with the pathway impact. The graph was obtained by plotting on the $y$-axis the $-\log _{10}$ transforms of $p$-values from the pathway enrichment analysis and on the $x$-axis the pathway impact values derived from the pathway topology analysis. The top three most significant pathways ( $p$-value $<0.05$; pathway impact $(\mathrm{pi})>0$ ) are depicted: purine metabolism $\left(p\right.$-value $\left.=1.3987 \times 10^{-6}, \mathrm{pi}=0.18529\right)$; phenylalanine, tyrosine, and tryptophan biosynthesis $(p$-value $=0.0039386$, $\mathrm{pi}=0.09009)$; zeatin biosynthesis $(p$-value $=0.025118 ; \mathrm{pi}=0.0271)$. The metabolomic analysis was repeated three times using different biological samples giving the same results.

Moreover, among the first top-ten significantly uniquely enriched pathways were "nicotinate and nicotinamide metabolism," "arginine biosynthesis," and "pentose phosphate pathway".

Purines are crucial components of nucleic acids and they play also an important role in metabolic regulation and energy storage. In plants, purines are also involved in nitrogen metabolism and in the formation of metabolic intermediates, among which is allantoin, that could also function in stress protection [38]. Studies in several plant species emphasized the correlation between endogenous allantoin levels and physiological responses to various stress, such as drought [39], nutrient deprivation [40] and high salinity [41]. Moreover, allantoin, and purines in general, enhance stress tolerance and protection by stimulating intermediate metabolites involved in stress responses [42]. Noteworthily, related to the fact that we found "purine metabolism" downregulated while "pyrimidine metabolism" was upregulated, we can mention that there are several endogenous and exogenous factors that affect the synthesis of purine and pyrimidine [26], and the accumulation of methylglyoxal could be one of the causes of the different behavior we found for the two pathways. In addition, we have also to consider that besides being the building blocks for DNA and RNA synthesis, purine and pyrimidine, are also precursors of energy sources, coenzymes, and primary and secondary compounds, whose syntheses can be up and/or downregulated depending on the contingent needs. Moreover, there are examples in the literature where the two pathways do not follow the same trend, supporting the reliability of the result [43]. Purines act also as precursors in the formation of zeatin, which plays a role in cell division, shoot formation, and adaptation to stress, as we will discuss later. Among 
the downregulated pathways is that of the aromatic amino acids phenylalanine, tyrosine, and tryptophan, which act as precursors of several secondary metabolites important for plant growth. Several lines of evidence suggest that a close relationship exists between cellular redox state and amino acid metabolism-in particular, that related to aromatic amino acids [44]. These amino acids are synthesized via the shikimate pathway and are essential precursors of the phenylpropanoid pathway, which is a great source of metabolites in plants [45]. Phenylpropanoids are required for diverse processes, among which are the lignin biosynthesis and flavonoid and coumarin formation [46]. Recently, flavonoids have been identified as regulators of GLYI and GLYII activity in tomato seedlings under salt stress [47]. In Arabidopsis, the phenylpropanoid pathway has been studied, especially for its correlation in defense and survival mechanisms [45]. These results could be linked to the compromised growth and defense in glyI4 mutant, as we previously observed [19]. Finally, another downregulated pathway highlighted in this study is that of zeatin biosynthesis. Zeatin is a phytohormone derived from purine metabolism, and it is a component of the family known as cytokinins (CKs). Several studies have shown that cytokinins have roles in plant growth, abiotic stress responses, pathogen and herbivore resistance, and cell division regulation [48]. A great correlation between GLYI and CKs has been documented in several species [47]. A genome-wide transcriptional study of $A$. thaliana ipt mutants (deficient in cytokinin) reported that GLYI expression is altered in mutants under stress. In particular, GLYI4 was fivefold upregulated in mutants versus wild-type under salt stress, probably due to a decreased activity of cytokinin [49]. In addition, it has been reported that a stunted growth occurs in stress conditions, due to the downregulation of the cytokinin pathway [50]. Therefore, this finding could also be linked to the reduced plant development and growth observed in the glyI4 mutant [19].

Taken together, the metabolite analysis highlighted that when GLYI4 is no longer functional, mechanisms related to plant growth and defense are likely to be affected, and metabolic cellular rewiring involves energy maintenance and redox-related processes.

\subsection{GLYI4 as a Potential Positive Regulator of Plant Growth and Defense}

The plant growth-defense trade-off is fundamental for optimizing plant performance and fitness in a changing biotic/abiotic environment. Looking at the results discussed in the previous paragraph, we found very intriguing the link between the downregulation of aromatic amino acids pathway and the shikimate/phenylpropanoid pathway, which is largely implicated in growth and defense. We then decided to check the expression of three valuable marker genes of the shikimate/phenylpropanoid pathway, phenylalanine ammonia lyase 2 (PAL2, At3g53260), cinnamyl alcohol dehydrogenase 4 (CAD4, At3g19450), and 5-enolpyruvylshikimate-3-phosphate synthase (EPSPS, At1g48860), in Col-8 and the glyI4 mutant. As shown in Figure 3, the three genes were significantly downregulated in the glyI4 mutant compared to Col-8.

It has frequently been observed that among secondary cell wall components, alterations in lignin biosynthesis result in changes in both growth and defense [51]. Lignin is an aromatic heteropolymer synthesized through an intricate phenylpropanoid metabolism. The phenylpropanoid pathway starts with the deamination of L-phenylalanine into cinnamic acid by PAL enzymes. The cinnamic acid undergoes a series of reduction processes in which the CAD enzymes are involved, to finally produce monolignols whose polymerization yields lignin [52]. Lignin is the second major structural component of the plant cell wall in supporting and conducting tissues, and it is used to reinforce mechanical strength and create a chemical and microbial resistant barrier [53]. Lignin, together with other chemical compounds such as suberin and phenols, is the first barrier in case of pathogen penetration [54-57]. For instance, host cell-induced lignin biosynthesis in response to fungal colonization has been reported in banana roots [58], wheat cells [59], and Camelina sativa $[52,60]$. In Arabidopsis, $C A D$, and $P A L$ genes have been found to act as essential components of defense against virulent and avirulent strains of the bacterial pathogen Pseudomonas syringae pv. tomato and as critical component in the resistance 
against abiotic stresses [61-63]. EPSPS catalyzes the transfer of the enolpyruvyl moiety of phosphoenolpyruvate (PEP) to the 5-hydroxyl of shikimate-3-phosphate (S3P) to produce enolpyruvyl shikimate-3-phosphate and inorganic phosphate. EPSPS is a key enzyme in the shikimate pathway, which is extremely important because about $35 \%$ or more plant biomass in dry matter form is represented by aromatic molecules derived directly from this pathway [64]. EPSPS is essential for the biosynthesis of aromatic amino acids, lignin, and secondary metabolites, including defensive compounds [65], suggesting that EPSPS is important for the survival, defense, growth, and development of plants. The involvement of this gene in plant growth is further corroborated by the evidence that Arabidopsis plants overexpressing EPSPS showed stimulated biosynthesis of auxin, an important plant growth hormone [66]. According to the stunted phenotype of glyI4 mutant, we can hypothesize that the downregulation of PAL2, CAD4, and EPSPS, compared to Col-8, could be one of the reasons for the impaired plant growth and defense [19].

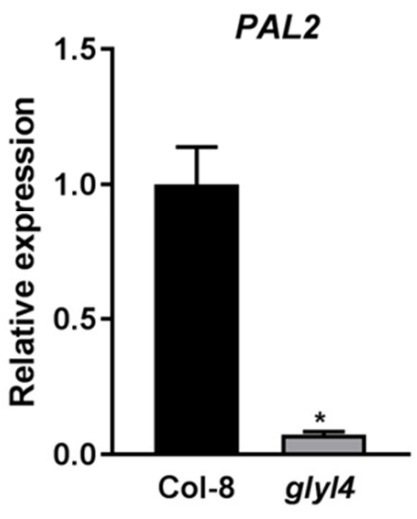

(a)

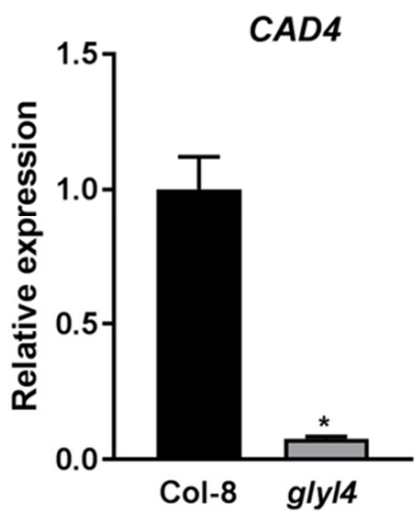

(b)

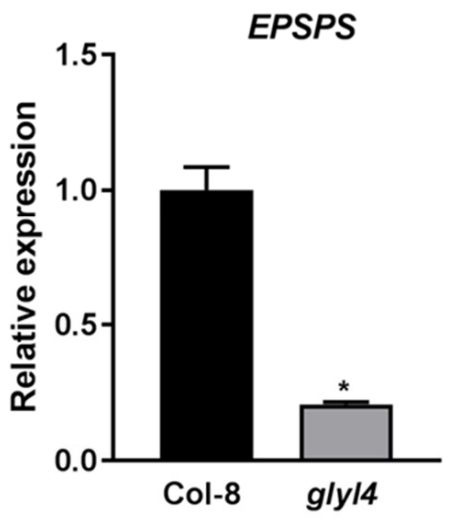

(c)

Figure 3. PAL2 (a), CAD4 (b), and EPSPS (c) transcript levels relative to the reference gene UBI10 in Col-8 and the glyI4 mutant. An asterisk indicates a statistically significant difference between genotypes (Mann-Whitney test $p$-value $<0.05)$. Error bars represent means $\pm \operatorname{SDs}(n=3)$.

\subsection{GLYI4 Affects Plant Antioxidant Responses}

ROS are produced in both unstressed and stressed cells. Plants have a well-developed defense systems against ROS, involving both limiting ROS formation and removing them. Under unstressed conditions, the formation and removal of ROS are well balanced. However, the defense system, when dealing with increased ROS formation under stressed conditions, can be overwhelmed. When this occurs, plants respond to high levels of ROS with increased enzymatic or non-enzymatic antioxidant processes [67]. In our previous work, we reported that glyI4 loss-of-function causes a great increase in ROS production and deprivation of GSH, likely due to MG accumulation [19]. In particular, using a ROSsensitive dye, a high level of $\mathrm{H}_{2} \mathrm{O}_{2}$ in Arabidopsis glyI4 leaves was highlighted [19]. To corroborate our previous results and to further validate the impact of glyI4 loss-of-function on redox processes, we investigated key components of the ROS pathway in the glyI4 mutant. In particular, we analyzed the activity of ROS scavenging enzymes such as superoxide dismutase (SOD) and catalase (CAT) and lipid peroxidation (by measuring thiobarbituric acid reactive substance assay (TBARS) content) in glyI4 leaves (Figure 4). 


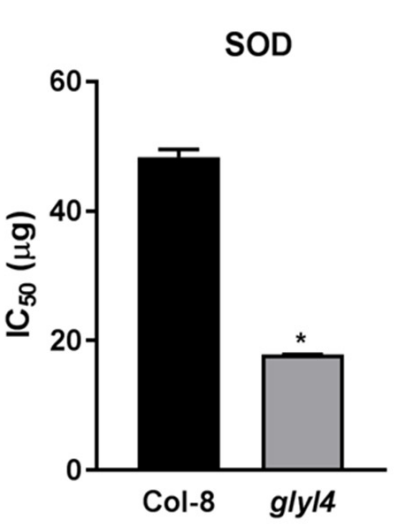

(a)

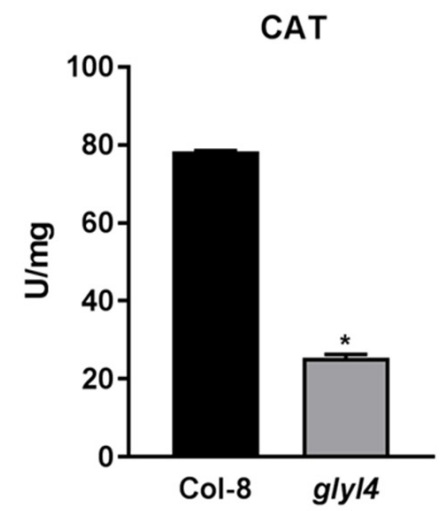

(b)

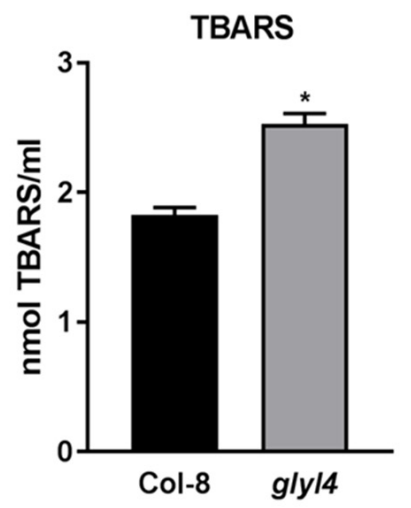

(c)

Figure 4. Antioxidant enzyme activities and lipid peroxidation in the glyI4 mutant. (a) superoxide dismutase (SOD); (b) catalase (CAT); (c) TBARS. Asterisks indicate statistically significant difference between genotypes. (Mann-Whitney test $p$-value $<0.05)$. Error bars represent means $\pm \operatorname{SDs}(n=3)$.

\subsubsection{Hydrogen Peroxide Is Not Efficiently Scavenged by Antioxidant Machinery}

Within a cell, the SODs constitute the first line of defense against ROS. The superoxide anion $\left(\mathrm{O}_{2}{ }^{-}\right)$is produced at any location where an electron transport chain is active, and hence $\mathrm{O}_{2}$ transformation may occur in different compartments of the cell [68], including mitochondria, chloroplasts, microsomes, glyoxysomes, peroxisomes, apoplasts, and the cytosol. Thus, it is not surprising to find that SODs are present in all these subcellular locations [67]. These enzymes belong to the family of metal-enzymes that catalyze the dismutation of superoxide anions $\left(\mathrm{O}_{2}{ }^{-}\right)$into molecular oxygen $\left(\mathrm{O}_{2}\right)$ and hydrogen peroxide $\left(\mathrm{H}_{2} \mathrm{O}_{2}\right)$. In our work, the IC50 of the glyI4 mutant was 2.5 times less than that of Col-8, suggesting that less protein extract is required to achieve $50 \%$ inhibition of formazan production. This means that SOD activity in the glyI4 mutant is greater than that in Col-8. High level of SOD activity was found in Citrus sinensis leaves where MG accumulation was reported [69]. Catalases (CATs) are heme-containing enzymes present in all organisms that play a central role in maintaining a balance of cellular hydrogen peroxide. The typical catalase reaction is the dismutation of two molecules of $\mathrm{H}_{2} \mathrm{O}_{2}$ to water and $\mathrm{O}_{2}$. $\mathrm{As}_{2} \mathrm{H}_{2}$ is relatively stable and present in several subcellular compartments, its impact is strongly dependent by the removal ability of the antioxidant system [70]. In our work, CAT activity decreased in the glyI4 mutant as compared to Col-8. Similarly, lower CAT protein levels were found in Citrus sinensis leaves that accumulated MG, compared to leaves where MG accumulation was lower. This might be related to the high sensitivity of CAT to oxidative stress [71,72] and to a reduced enzyme production under stress which keeps CAT levels low [69]. According to our results, we can speculate that $\mathrm{H}_{2} \mathrm{O}_{2}$ production driven by SOD is not scavenged by CAT, whose activity is compromised by MG accumulation.

\subsection{2. glyI4 Mutant Suffers of Lipid Peroxidation}

Lipid peroxidation is a deleterious process in plants, which affects membrane properties, causes protein degradation, and limits ion transport capacity, ultimately triggering the cell death process [73]. One of the most commonly used methods to detect lipid peroxidation is the TBARS assay. The assay involves the reaction of lipid peroxidation products, primarily malondialdehyde (MDA), with thiobarbituric acid (TBA), which leads to the formation of MDA-TBA2 adducts called TBARS. TBARS produce a red-pink color that can be measured spectrophotometrically at $532 \mathrm{~nm}$. The highest TBARS content was found in the glyI4 mutant, suggesting a higher level of oxidative stress in these plants, probably due to an impaired detoxification system. In addition, it has been reported that over-accumulation of MG can lead to lipid peroxidation in plant cells [35,74]. In particular, Yadav et al. [35] reported that the salt-induced increases of MG and MDA concentrations were much lower in transgenic tobacco plants overexpressing GLYI and/or GLYII than in wild-type plants. 
Taken together, our results corroborate the great impact of MG accumulation on ROS accumulation and scavenging.

\section{Materials and Methods}

\subsection{Plant Material and Growth Conditions}

The A. thaliana T-DNA line in Col-8 background glyI4 (AGI: At1g15380) was purchased from NASC (http:/ /arabidopsis.info/, 20 August 2014), available with the ID: SALK_067593C. The same mutant was already tested and used for experiments $[18,19]$. Arabidopsis glyI4 and Col-8 seeds were sown in cultivation containers filled with autoclaved river sand. Sand was supplied with half-strength Hoagland solution (Sigma, Steinheim, Germany). To achieve a high relative humidity for germination, cultivation containers were enclosed in a tray with water and covered with a transparent lid. Seeds were stratified for 2 days at $4{ }^{\circ} \mathrm{C}$ in the dark to ensure a homogeneous germination after which the tray was moved to a growth chamber with an 8-h day /16-h night rhythm, a temperature of $21{ }^{\circ} \mathrm{C}$, and a light intensity of $100 \mu \mathrm{mol} \mathrm{m}{ }^{-2} \mathrm{~s}^{-1}$. After 8 days, the lids of the trays were slightly opened and gradually removed over a two-day-period. Ten-day-old seedlings were transplanted into individual pots filled with autoclaved river sand and potting soil mixture $(1: 1, v: v)$. Pots were supplied with water from the bottom up three times per week. At an age of 3 weeks, the plants were supplied with half-strength Hoagland solution once a week. Five-week-old plants were used for all experiments. Plants growth was repeated three times independently and each plant set represented one biological replicate.

\subsection{Metabolite Extraction and LC-MS Analysis}

Two hundred milligrams of Col-8 and glyI4 leaves were finely ground in liquid nitrogen, and powder was used for metabolite extractions. Cells were lysed by thermal shock (freezing/heating). A cold $\left(-20{ }^{\circ} \mathrm{C}\right)$ solution of $60 \%$ methanol $/ 40 \%$ chloroform was added to each tube. The tubes were mixed for $30 \mathrm{~min}$ and subsequently centrifuged at $1000 \times g$ for $1 \mathrm{~min}$ at $4{ }^{\circ} \mathrm{C}$, before being transferred to $-20^{\circ} \mathrm{C}$ for $2-8 \mathrm{~h}$. After thawing, liquid phases were recovered, and the samples were incubated at $4{ }^{\circ} \mathrm{C}$ for $20 \mathrm{~min}$ and centrifuged at $13,500 \times g$ for $10 \mathrm{~min}$ at $4{ }^{\circ} \mathrm{C}$; and the collected supernatants were dried to get visible pellets. Lastly, the dried samples were resuspended in water and $5 \%$ formic acid, and transferred to glass autosampler vials for LC/MS analysis. Twenty microliters of samples were injected into an Ultra High-Performance Liquid Chromatography (UHPLC) system (Ultimate 3000, Thermo Fisher Scientific, Waltham, MA, USA) and run in positive ion mode. A Reprosil C18 column $(2.0 \mathrm{~mm} \times 150 \mathrm{~mm}, 2.5 \mu \mathrm{m}$ - Dr Maisch HPLC GmbH, Ammerbuch, Germany) was used for metabolite separation. Chromatographic separations were achieved at a column temperature of $30^{\circ} \mathrm{C}$ and flow rate of $0.2 \mathrm{~mL} / \mathrm{min}$. A $0-100 \%$ linear gradient of solvent $\mathrm{A}$ ( $\mathrm{ddH}_{2} \mathrm{O}, 0.1 \%$ formic acid) to $\mathrm{B}$ (acetonitrile, $0.1 \%$ formic acid) was employed over $20 \mathrm{~min}$, returning to $100 \% \mathrm{~A}$ in $2 \mathrm{~min}$ and a 6-min post-time solvent $\mathrm{A}$ hold. The UHPLC system was coupled online with a mass spectrometer Q-Exactive (Thermo Fisher Scientific, Waltham, MA, USA) scanning in full MS mode ( $2 \mu$ scans) at 70,000 resolution in the 67 to $1000 \mathrm{~m} / \mathrm{z}$ range, target of $1 \times 10^{6}$ ions and a maximum ion injection time (IT) of $35 \mathrm{~ms}$. Source ionization parameters were: spray voltage, $3.8 \mathrm{kV}$; capillary temperature, $300{ }^{\circ} \mathrm{C}$; sheath gas, 40; auxiliary gas, 25; S-Lens level, 45 . Calibration was performed before each analysis against positive ion mode calibration mixes (Piercenet, Thermo Fisher Scientific, Waltham, MA, USA) to ensure sub ppm error of the intact mass. Three biological and technical replicates were carried out for both the samples.

\subsection{Metabolomic Data Processing}

\subsubsection{Data Elaboration and Statistical Analysis}

Raw files of metabolomics data from technical and biological replicates of samples were analyzed using XCMS Online, a freely accessible metabolite database called METLIN (http://metlin.scripps.edu, 10 July 2019) [75]. Raw data sets were uploaded to XCMS Online, and a single group job was created to analyze the difference between profile of glyI4 
mutant versus Col-8. The raw data files were then processed for peak detection, retentiontime correction, chromatogram alignment, metabolite feature metadata, and statistical evaluation using the predefined workflow settings for Orbitrap, and metabolite identification was facilitated through METLIN standard database matching and Kyoto Encyclopedia of Genes and Genomes (KEGG) pathway database. XCMS extracted metabolomic features with statistically significant expression changes among the two groups to produce a list of differentially expressed metabolites based on $\log _{2}$-fold change $>|1|$ and $p$-value $<0.05$.

\subsubsection{Pathway Analysis}

Metabolite data set were processed using the MetaboAnalyst 5.0 software (http:/ / www.metaboanalyst.ca, 20 October 2021) [76]. The list of metabolites was imported into Metaboanalyst and mapped to the Kyoto Encyclopedia of Genes and Genomes (KEGG) in the "Pathway analysis" tool that supports metabolic pathway analysis, integrating pathway enrichment analysis and pathway topology analysis. Metabolic pathways with $p$-values $<0.05$ and pathway impact $>0$ were considered significantly enriched. Among these pathways, the top three most significant were widely discussed.

\subsection{RNA Extraction and RT-qPCR}

RNA was isolated from Col-8 and glyI4 leaves using the "Nucleospin RNA II" kit (Machery-Nagel, Düren, Germany) according to the manufacturer's instructions. The RNA quality and concentration were estimated by agarose gel electrophoresis and spectrophotometric reading, respectively. DNA contamination was assessed by detecting the absence of amplification bands in no RT samples. cDNA was synthesized using the ImProm-II ${ }^{\mathrm{TM}}$ Reverse Transcription System (Promega, Madison, WI, USA) starting from $1 \mu \mathrm{g}$ of RNA as template and using the oligo-dT primer for first strand synthesis. Diluted cDNA was used as template for Real-Time qPCR with a CFX 96 Touch Real-Time PCR detection system (Bio-Rad, Hercules, CA, USA). Each $10 \mu \mathrm{L}$ reaction mix contained $5 \mu \mathrm{L}$ Sso Advanced SYBR Green Supermix (Bio-Rad) and $0.5 \mu \mathrm{M}$ (final concentration) gene specific primers. Primers were designed with Primer3 software (http:/ / bioinfo.ut.ee/primer3-0.4.0/, 20 May 2019) under default conditions. The amplification program was as follows: $95^{\circ} \mathrm{C}$ for $5 \mathrm{~s} ; 40$ cycles at $95{ }^{\circ} \mathrm{C}$ for $5 \mathrm{~s}$ and primers annealing at $54{ }^{\circ} \mathrm{C}$ for $45 \mathrm{~s}$. To detect and exclude non-specific amplicons, the melting curves of all PCR products were analyzed $\left(65-95^{\circ} \mathrm{C}\right.$ with an increase of $0.5^{\circ} \mathrm{C}$ every $5 \mathrm{~s}$ ). Real-time DNA amplification was processed using CFX Manager ${ }^{\mathrm{TM}}$ Soft-

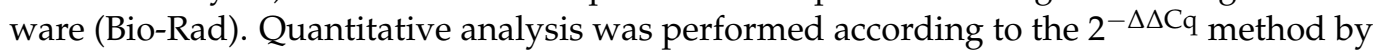
applying efficiency correction formula. The normalized amount of target reflects the relative amount of target transcripts with respect to the endogenous reference gene UBI10. Gene expression fold change was calculated in glyI4 mutant relative to Col-8 plants. The Arabidopsis gene identifier (AGI) numbers of the studied genes are AT3g53260 (PAL2), AT3g19450 (CAD4), AT1g48860 (EPSPS), At4g05320 (UBI10). Primers were the following: PAL2 (Fw: AGGCAGCGTTAAGGTTGAGT; Rv: GGTGACTCCGTAACTGTCAGTAC), CAD4 (Fw: GACACCATGATCGTCAATCAAAAG; Rv: TCAACGGACTATAAACCGTTACTC), EPSPS (Fw: TGCTAAATGGTTCTGAGATTCGTC; Rv: AGACCCGAGATTTCTCTAAT GGG), UBI10 (Fw: AAAGAGATAACAGGAACGGAAACATAGT; Rv: GGCCTTGTAT AATCCCTGATGAATAAG). Results of qPCR analyses were verified by three independent experiments each of them having three technical replicates.

\subsection{Protein Extraction}

One gram of Col-8 or glyI4 leaves, from plants grown as described in 3.1, were finely ground with mortar and pestle with continuous addition of liquid nitrogen. The powder was resuspended in $5 \mathrm{~mL}$ of a cold extraction buffer consisting of $50 \mathrm{mM}$ sodium phosphate buffer (pH 7.5) including $1 \mathrm{mM}$ EDTA, 1\% (w/v) polyvinylpyrrolidone, $3 \mathrm{mM}$ DTT, and a cocktail of protease inhibitors (Complete ULTRA tablets, Roche, Basel, Switzerland). The homogenate was centrifuged (Universal 32R, Hettich, Tuttlingen, Germany) at $9000 \mathrm{rpm}$ for $15 \mathrm{~min}$ at $4{ }^{\circ} \mathrm{C}$ and the supernatant was used for enzyme activity assays as described 
in [77]. The protein content was estimated according to Bradford method using BSA as a standard.

\subsection{Superoxide Dismutase Activity Assay}

SOD activity was assayed with the SOD determination kit (Sigma-Aldrich, Uppsala, Sweden) following the manufacturer's instructions. The SOD Assay Kit is based on the use of Dojindo's highly water-soluble tetrazolium salt (2-(4-iodophenyl)-3-(4-nitrophenyl)-5(2,4-disulfophenyl)-2H tetrazolium, monosodium salt), which produces a water-soluble formazan dye upon reduction with a superoxide anion. The rate of the reduction is linearly related to the xanthine oxidase activity, and it is inhibited by SOD. Since the absorbance at $440 \mathrm{~nm}$ is proportional to the amount of formazan dye, the SOD activity can be quantified as an inhibition activity by measuring the decrease in the color development at $440 \mathrm{~nm}$. Thus, inhibition activity corresponds to the amount of total protein extract necessary to reduce the formation of formazan by $50 \%\left(\mathrm{IC}_{50}\right)$.

\subsection{Catalase Activity Assay}

CAT activity was measured by monitoring the decrease of absorbance at $240 \mathrm{~nm}$, $25^{\circ} \mathrm{C}$, due to the decomposition of $\mathrm{H}_{2} \mathrm{O}_{2}\left(\varepsilon_{\mathrm{mM}}=0.0436 \mathrm{mM}^{-1} \mathrm{~cm}^{-1}\right)$. The reaction mixture (1 mL final volume) contained $19 \mathrm{mM}$ hydrogen peroxide $\left(\mathrm{H}_{2} \mathrm{O}_{2}\right)$ in $50 \mathrm{mM}$ potassium phosphate buffer ( $\mathrm{pH}$ 7.0); the reaction was started by adding $50 \mu \mathrm{g}$ quantity of protein extract. Catalase specific activity is defined in terms of $\mu$ moles of hydrogen peroxide consumed per minute per $\mathrm{mg}$ of protein sample.

\subsection{Thiobarbituric Acid Reactive Substance Measurement}

The level of TBARS was used to assess lipid peroxidation following the protocol described in [77]. Briefly, four hundred milligrams of frozen leaves were finely ground using a mortar and pestle under continuous addition of liquid nitrogen. The powder was resuspended in $3 \mathrm{~mL}$ of trichloroacetic acid (TCA), $0.1 \%$, and mixed on the vortex until homogenized. Following centrifugation at 13,000 rpm for $10 \mathrm{~min}, 400 \mu \mathrm{L}$ of the supernatant (or $0.1 \%$ TCA for the blank) was added either to $1 \mathrm{~mL}$ of $0.5 \%$ TBA in $20 \%$ TCA (+TBA solution) or to $1 \mathrm{~mL}$ of $20 \%$ TCA (-TBA solution) (dilution factor 1:3.5). Samples were incubated at $80^{\circ} \mathrm{C}$ for $30 \mathrm{~min}$ and then cooled on ice. After centrifugation at $13,500 \mathrm{rpm}$ for $5 \mathrm{~min}$, the absorbance was measured both at $532 \mathrm{~nm}$, which represents the maximum absorbance of the TBA-TBARS complex, and at $600 \mathrm{~nm}$ to allow correction of non-specific turbidity. To calculate the TBARS equivalent $\left(\mathrm{nmol} \mathrm{mL} \mathrm{L}^{-1}\right)$, the $\varepsilon_{\mu \mathrm{M}}\left(0.155 \mu \mathrm{M}^{-1} \mathrm{~cm}^{-1}\right)$ of malondialdehyde (MDA), one of the main products of membrane damage, was used according to the following formula:

$$
\left[\mathrm{A} / \varepsilon_{\mu \mathrm{M}} \mathrm{MDA}\right] \times \text { dilution factor }
$$

where $\mathrm{A}=\left[\left(\mathrm{A}_{532} \mathrm{~nm}_{(+\mathrm{TBAsol})}-\mathrm{A}_{600} \mathrm{~nm}_{(+\mathrm{TBAsol})}\right)-\left(\mathrm{A}_{532} \mathrm{~nm}_{(-\mathrm{TBAsol})}-\mathrm{A}_{600} \mathrm{~nm}(-\mathrm{TBAsol})\right)\right]$

\subsection{Statistical Analysis}

A Mann-Whitney non-parametric test was performed with GraphPad Prism 7.0 (GraphPad Software Inc., San Diego, CA, USA). In detail, the test was applied to analyze differences in PAL2, CAD4, and EPSPS transcript levels in Col-8 and glyI4 plants (par. 2.2), and to analyze differences in SOD and CAT activity, and T-BARS content in Col-8 and glyI4 plants (par. 2.3).

\section{Conclusions}

Our current work has shown that metabolic processes linked to redox reactions and the generation of energy sources are upregulated in the glyI4 mutant compared to Col-8, whereas those involved in defense and growth are downregulated. These findings strongly matched the previously results obtained with the glyI4 mutant, in which MG rising led to ROS accumulation, reduced growth and fitness, and compromised defense [19]. Lastly, 
these results have also demonstrated that metabolic profiling gives important information essential to understanding physiological and biochemical rewiring, and ultimately to shedding some light on how these changes affect plant phenotypes. In the future, the awareness of GLYI4 as a novel potential player in the growth-defense trade-off could be exploited to develop sustainable crops.

Supplementary Materials: The following are available online at https://www.mdpi.com/article/10 .3390 / plants10112464/s1, Table S1: All detected and differentially synthesized metabolites in glyI4 mutant compared to Col-8 ( $\log _{2}$-fold change $>|1|$, $p$-value $\left.<0.05\right)$.

Author Contributions: Conceptualization, S.P. and C.C.; methodology, S.P., L.B., G.S.F. and A.M.T.; validation, S.P., G.S.F. and L.B.; formal analysis, S.P., L.B., G.S.F., I.B. and A.M.T.; investigation, S.P., L.B. and C.C.; resources, C.C.; data curation, S.P., L.B. and G.S.F.; writing-original draft preparation, S.P., G.S.F.; writing-review and editing, S.P., L.B. and C.C.; supervision, C.C.; project administration, C.C.; funding acquisition, S.P. All authors have read and agreed to the published version of the manuscript.

Funding: This research was funded by MIUR (Ministry for Education, University and Research) MIUR- PRIN 2017, grant number PROSPECT 2017JLN833_005.

Institutional Review Board Statement: Not applicable.

Informed Consent Statement: Not applicable.

Data Availability Statement: The raw metabolomics data are available in the EMBL-EBI MetaboLights database (DOI:10.1093/nar/gkz1019, PMID:31691833) with the identifier MTBLS3724.

Conflicts of Interest: The authors declare no conflict of interest. The funders had no role in the design of the study; in the collection, analyses, or interpretation of data; in the writing of the manuscript, or in the decision to publish the results.

\section{References}

1. Pieterse, C.M.J.; Van der Does, D.; Zamioudis, C.; Leon-Reyes, A.; VanWees, S.C.M. Hormonal modulation of plant immunity. Annu. Rev. Cell Dev. Biol. 2012, 28, 489-521. [CrossRef]

2. Rejeb, I.B.; Pastor, V.; Mauch-Mani, B. Plant responses to simultaneous biotic and abiotic stress: Molecular mechanisms. Plants 2014, 3, 458-475. [CrossRef]

3. Pieterse, C.M.J.; Leon-Reyes, A.; Van der Ent, S.; Van Wees, S.C.M. Networking by small-molecule hormones in plant immunity. Nat. Chem. Biol. 2009, 5, 308-316. [CrossRef] [PubMed]

4. Yang, L.; Wen, K.S.; Ruan, X.; Zhao, Y.X.; Wei, F.; Wang, Q. Response of plant secondary metabolites to environmental factors. Molecules 2018, 23, 762. [CrossRef]

5. Brilli, F.; Loreto, F.; Baccelli, I. Exploiting Plant Volatile Organic Compounds (VOCs) in agriculture to improve sustainable defense strategies and productivity of crops. Front. Plant Sci. 2019, 10, 264. [CrossRef] [PubMed]

6. Hoque, T.S.; Okuma, E.; Uraji, M.; Furuichi, T.; Sasaki, T.; Hoque, M.A.; Nakamura, Y.; Murata, Y. Inhibitory effects of methylglyoxal on light-induced stomatal opening and inward $\mathrm{K}+$ channel activity in Arabidopsis. Biosci. Biotechnol. Biochem. 2012, 76, 617-619. [CrossRef]

7. Hoque, T.S.; Uraji, M.; Tuya, A.; Nakamura, Y.; Murata, Y. Methylglyoxal inhibits seed germination and root elongation and up-regulates transcription of stress-responsive genes in ABA-dependent pathway in Arabidopsis. Plant Biol. 2012, 14, 854-858. [CrossRef]

8. Hoque, T.S.; Uraji, M.; Ye, W.; Hossain, M.A.; Nakamura, Y.; Murata, Y. Methylglyoxal-induced stomatal closure accompanied by peroxidase mediated ROS production in Arabidopsis. Plant Physiol. 2012, 169, 979-986. [CrossRef]

9. Hoque, M.A.; Uraji, M.; Banu, M.N.A.; Mori, I.C.; Nakamura, Y.; Murata, Y. Methylglyoxal inhibition of cytosolic ascorbate peroxidase from Nicotiana tabacum. J. Biochem. Mol. Toxicol. 2012, 26, 315-321. [CrossRef]

10. Mano, J. Reactive carbonyl species: Their production from lipid peroxides, action in environmental stress, and the detoxification mechanism. Plant Physiol. Biochem. 2012, 59, 90-97. [CrossRef] [PubMed]

11. Hoque, T.S.; Hossain, M.A.; Mostofa, M.G.; Burritt, D.J.; Fujita, M.; Tran, L.-S.P. Methylglyoxal: An emerging signaling molecule in plant abiotic stress responses and tolerance. Front. Plant Sci. 2016, 7, 1341. [CrossRef]

12. Singla-Pareek, S.L.; Kaur, C.; Kumar, B.; Pareek, A.; Sopory, S.K. Reassessing plant glyoxalases: Large family and expanding functions. New Phytol. 2020, 227, 714-721. [CrossRef]

13. Sankaranarayanan, S.; Jamshed, M.; Kumar, A.; Skori, L.; Scandola, S.; Wang, T.; Spiegel, D.; Samuel, M.A. Glyoxalase goes green: The expanding roles of glyoxalase in plants. Int. J. Mol. Sci. 2017, 18, 898. [CrossRef] 
14. Kaur, C.; Singla-Pareek, S.L.; Sopory, S.K. Glyoxalase and methylglyoxal as biomarkers for plant stress tolerance. Crit. Rev. Plant Sci. 2014, 33, 429-456. [CrossRef]

15. Gupta, B.K.; Sahoo, K.K.; Ghosh, A.; Tripathi, A.K.; Anwar, K.; Das, P.; Singh, A.K.; Pareek, A.; Sopory, S.K.; Singla-Pareek, S.L. Manipulation of glyoxalase pathway confers tolerance to multiple stresses in rice. Plant Cell Environ. 2018, 41, 1186-1200. [CrossRef]

16. Li, T.; Cheng, X.; Wang, X.; Li, G.; Wang, B.; Wang, W.; Zhang, N.; Han, Y.; Jiao, B.; Wang, Y.; et al. Glyoxalase I-4 functions downstream of NAC72 to modulate downy mildew resistance in grapevine. Plant J. 2021. [CrossRef] [PubMed]

17. Mustafiz, A.; Singh, A.K.; Pareek, A.; Sopory, S.K.; Singla-Pareek, S.L. Genome-wide analysis of rice and Arabidopsis identifies two glyoxalase genes that are highly expressed in abiotic stresses. Funct. Integr. Genom. 2011, 11, 293-305. [CrossRef] [PubMed]

18. Proietti, S.; Caarls, L.; Coolen, S.; Van Pelt, J.A.; Van Wees, S.C.M.; Pieterse, C.M.J. Genome-wide association study reveals novel players in defense hormone crosstalk in Arabidopsis. Plant Cell Environ. 2018, 41, 2342-2356. [CrossRef] [PubMed]

19. Proietti, S.; Falconieri, G.S.; Bertini, L.; Baccelli, I.; Paccosi, E.; Belardo, A.; Timperio, A.M.; Caruso, C. GLYI4 plays a role in methylglyoxal detoxification and jasmonate-mediated stress responses in Arabidopsis thatiana. Biomolecules 2019, 9, 635. [CrossRef] [PubMed]

20. Fraire-Velázquez, S.; Balderas-Hernández, V.E. Abiotic Stress in Plants and Metabolic Responses, Abiotic Stress-Plant Responses and Applications in Agriculture, Kourosh Vahdati and Charles Leslie; IntechOpen: London, UK, 2013. [CrossRef]

21. Caldana, C.; Degenkolbe, T.; Cuadros-Inostroza, A.; Klie, S.; Sulpice, R.; Leisse, A.; Steinhauser, D.; Fernie, A.R.; Willmitzer, L.; Hannah, M.A. High-density kinetic analysis of the metabolomic and transcriptomic response of Arabidopsis to eight environmental conditions. Plant J. 2011, 67, 869-884. [CrossRef]

22. Schrimpe-Rutledge, A.C.; Codreanu, S.G.; Sherrod, S.D.; McLean, J.A. Untargeted metabolomics strategies-challenges and emerging directions. J. Am. Soc. Mass Spectr. 2016, 27, 1897-1905. [CrossRef] [PubMed]

23. Braun, J.D.; Pastene, D.O.; Breedijk, A.; Rodriguez, A.; Hofmann, B.B.; Sticht, C.; von Ochsenstein, E.; Allgayer, H.; van den Born, J.; Bakker, S.; et al. Methylglyoxal down-regulates the expression of cell cycle associated genes and activates the p53 pathway in human umbilical vein endothelial cells. Sci. Rep. 2019, 9, 1152. [CrossRef] [PubMed]

24. Suzuki-Yamamoto, M.; Mimura, T.; Ashihara, H. Effect of short-term salt stress on the metabolic profiles of pyrimidine, purine and pyridine nucleotides in cultured cells of the mangrove tree, Bruguiera sexangula. Physiol. Plant 2006, 128, 405-414. [CrossRef]

25. Bolt, R.; Zrenner, R. Purine and pyrimidine biosynthesis in higher plants. Physiol. Plant 2002, 117, 297-304. [CrossRef]

26. Stasolla, C.; Katahira, R.; Thorpe, T.A.; Ashihara, H. Purine and pyrimidine nucleotide metabolism in higher plants. J. Plant Physiol. 2003, 160, 1271-1295. [CrossRef]

27. Fang, J.; Uchiumi, T.; Yagi, M.; Matsumoto, S.; Amamoto, R.; Takazaki, S.; Yamaza, H.; Nonaka, K.; Kang, D. Dihydro-orotate dehydrogenase is physically associated with the respiratory complex and its loss leads to mitochondrial dysfunction. Biosci. Rep. 2013, 33, e00021. [CrossRef]

28. Handy, D.E.; Loscalzo, J. Redox Regulation of Mitochondrial Function. Antioxid. Redox Signal. 2012, 16, 1323-1367. [CrossRef]

29. Schmidt, B.; de Assis, A.M.; Battu, C.E.; Rieger, D.K.; Hansen, F.; Sordi, F.; Longoni, A.; Hoefel, A.L.; Farina, M.; Gonçalves, C.A.; et al. Effects of glyoxal or methylglyoxal on the metabolism of amino acids, lactate, glucose and acetate in the cerebral cortex of young and adult rats. Brain Res. 2010, 1315, 19-24. [CrossRef]

30. Slocum, R.D. Genes, enzymes and regulation of arginine biosynthesis in plants. Plant Physiol. Biochem. 2005, 43, 729-745. [CrossRef]

31. Winter, G.; Todd, C.D.; Trovato, M.; Forlani, G.; Funck, D. Physiological implications of arginine metabolism in plants. Front. Plant Sci. 2015, 6, 534. [CrossRef] [PubMed]

32. Nikiforova, V.; Kempa, S.; Zeh, M.; Maimann, S.; Kreft, O.; Casazza, A.P.; Riedel, K.; Tauberger, E.; Hoefgen, R.; Hesse, H. Engineering of cysteine and methionine biosynthesis in potato. Amino Acids 2002, 22, 259-278. [CrossRef] [PubMed]

33. Romero, L.C.; Aroca, M.Á.; Laureano-Marín, A.M.; Moreno, I.; García, I.; Gotor, C. Cysteine and cysteine-related signaling pathways in Arabidopsis thaliana. Mol. Plant 2014, 7, 264-276. [CrossRef]

34. Hell, R.; Wirtz, M. Molecular biology, biochemistry and cellular physiology of cysteine metabolism in Arabidopsis thaliana. In The Arabidopsis Book; American Society of Plant Biologists: Rockville, MD, USA, 2011; Volume 9, p. e0154. [CrossRef]

35. Yadav, S.K.; Singla-Pareek, S.L.; Ray, M.; Reddy, M.K.; Sopory, S.K. Transgenic tobacco plants overexpressing glyoxalase enzymes resist an increase in methylglyoxal and maintain higher reduced glutathione levels under salinity stress. FEBS Lett. 2005, 579, 6265-6271. [CrossRef] [PubMed]

36. Kusano, M.; Fukushima, A.; Redestig, H.; Kobayashi, M.; Otsuki, H.; Onouchi, H.; Naito, S.; Hirai, M.Y.; Saito, K. Comparative metabolomics charts the impact of genotype-dependent methionine accumulation in Arabidopsis thaliana. Amino Acids 2010, 39, 1013-1021. [CrossRef]

37. Kirma, M.; Araújo, W.L.; Fernie, A.R.; Galili, G. The multifaceted role of aspartate-family amino acids in plant metabolism. J. Exp. Bot. 2012, 63, 4995-5001. [CrossRef] [PubMed]

38. Werner, A.K.; Witte, C.-P. The biochemistry of nitrogen mobilization: Purine ring catabolism. Trends Plant Sci. 2011, 16, 381-387. [CrossRef]

39. Yobi, A.; Wone, B.W.M.; Xu, W.; Alexander, D.C.; Guo, L.; Ryals, J.A.; Olivier, M.J.; Cushman, J.C. Metabolomic profiling in Selaginella lepidophylla at various hydration states provides new insights into the mechanistic basis of desiccation tolerance. Mol. Plant 2013, 6, 369-385. [CrossRef] 
40. Rose, M.T.; Rose, T.J.; Pariasca-Tanaka, J.; Yoshihashi, T.; Neuweger, H.; Goesmann, A.; Frei, M.; Wissuwa, M. Root metabolic response of rice (Oryza sativa L.) genotypes with contrasting tolerance to zinc deficiency and bicarbonate excess. Planta 2012, 236, 959-973. [CrossRef]

41. Kanani, H.; Dutta, B.; Klapa, M.I. Individual vs. combinatorial effect of elevated $\mathrm{CO}_{2}$ conditions and salinity stress on Arabidopsis thaliana liquid cultures: Comparing the early molecular response using time-series transcriptomic and metabolomic analyses. BMC Syst. Biol. 2010, 4, 177. [CrossRef]

42. Watanabe, S.; Kounosu, Y.; Shimada, H.; Sakamoto, A. Arabidopsis xanthine dehydrogenase mutants defective in purine degradation show a compromised protective response to drought and oxidative stress. Plant Biotechnol. 2014, 31, 173-178. [CrossRef]

43. Misra, B.B.; de Armas, E.; Tong, Z.; Chen, S. Metabolomic responses of guard cells and mesophyll cells to bicarbonate. PLoS ONE 2015, 10, e0144206. [CrossRef]

44. Vivancos, P.D.; Driscoll, S.P.; Bulman, C.A.; Ying, L.; Emami, K.; Treumann, A.; Mauve, C.; Noctor, G.; Foyer, C.H. Perturbations of amino acid metabolism associated with glyphosate-dependent inhibition of shikimic acid metabolism affect cellular redox homeostasis and alter the abundance of proteins involved in photosynthesis and photorespiration. Plant Physiol. 2011, 157, 256-268. [CrossRef]

45. Tzin, V.; Galili, G. The biosynthetic pathways for shikimate and aromatic amino acids in Arabidopsis thaliana. Arab. Book 2010, 8, e0132. [CrossRef]

46. Fraser, C.M.; Chapple, C. The Phenylpropanoid Pathway in Arabidopsis. Arab. Book 2011, 9, e0152. [CrossRef]

47. Garai, S.; Bhowal, B.; Kaur, C.; Singla-Pareek, S.L.; Sopory, S.K. What signals the glyoxalase pathway in plants? Physiol. Mol. Biol. Plants 2021. [CrossRef]

48. Kieber, J.J.; Schaller, G.E. Cytokinin signaling in plant development. Development 2018, 145, dev149344. [CrossRef] [PubMed]

49. Nishiyama, R.; Le, D.T.; Watanabe, Y.; Matsui, A.; Tanaka, M.; Seki, M.; Yamaguchi-Shinozaki, K.; Shinozaki, K.; Phan Tran, L.-S. Transcriptome analyses of a salt-tolerant cytokinin-deficient mutant reveal differential regulation of salt stress response by cytokinin deficiency. PLoS ONE 2012, 7, e32124. [CrossRef]

50. Schäfer, M.; Brütting, C.; Canales, I.M.; Großkinsky, D.K.; Vankova, R.; Baldwin, I.T.; Meldau, S. The role of cis-zeatin-type cytokinins in plant growth regulation and mediating responses to environmental interactions. J. Exp. Bot. 2015, 66, 4873-4884. [CrossRef] [PubMed]

51. Xie, M.; Zhang, J.; Tschaplinski, T.J.; Tuskan, G.A.; Chen, J.-G.; Muchero, W. Regulation of lignin biosynthesis and its role in growth-defense tradeoffs. Front. Plant Sci. 2018, 9, 1427. [CrossRef]

52. Govender, N.T.; Mahmood, M.; Seman, I.A.; Wong, M.-Y. The phenylpropanoid pathway and lignin in defense against Ganoderma boninense colonized root tissues in Oil Palm (Elaeis guineensis Jacq.). Front. Plant Sci. 2017, 8, 1395. [CrossRef]

53. Liu, Q.; Luo, L.; Zheng, L. Lignins: Biosynthesis and biological functions in plants. Int. J. Mol. Sci. 2018, 19, 335. [CrossRef] [PubMed]

54. Boudet, A. Lignins and lignification: Selected issues. Plant Physiol. Biochem. 2000, 38, 81-96. [CrossRef]

55. Donaldson, L.A. Lignification and lignin topochemistry-An ultrastructural view. Phytochemistry 2001, 57, 859-873. [CrossRef]

56. Boerjan, W.; Ralph, J.; Baucher, M. Lignin biosynthesis. Annu. Rev. Plant Biol. 2003, 54, 519-546. [CrossRef] [PubMed]

57. Vanholme, R.; Morreel, K.; Ralph, J.; Boerjan, W. Lignin engineering. Curr. Opin. Plant Biol. 2008, 11, 278-285. [CrossRef] [PubMed]

58. Ana, R.D.; Ian, A.D. Panama disease: Cell wall reinforcement in banana roots in response to elicitors from Fusarium oxysporum f. sp. cubense race four. Am. Phytopathol. Soc. 2000, 90, 1173-1180. [CrossRef]

59. Tiburzy, R.; Reisener, H.J. Resistance of wheat to Puccinia graminis f.sp. tritici: Association of the hypersensitive reaction with the cellular accumulation of lignin-like material and callose. Physiol. Mol. Plant Pathol. 1990, 36, 109-120. [CrossRef]

60. Eynck, C.; Seguin-swartz, G.; Clarke, W.E.; Parkin, I.A.P. Monolignol biosynthesis is associated with resistance to Sclerotinia sclerotiorum in Camelina sativa. Mol. Plant Pathol. 2012, 13, 887-899. [CrossRef]

61. Tronchet, M.; Balagué, C.; Kroj, T.; Jouanin, L.; Roby, D. Cinnamyl alcohol dehydrogenases-C and D, key enzymes in lignin biosynthesis, play an essential role in disease resistance in Arabidopsis. Mol. Plant Pathol. 2010, 11, 83-92. [CrossRef]

62. Wu, Z.; Han, S.; Zhou, H.; Tuang, Z.K.; Wang, Y.; Jin, Y.; Shi, H.; Yang, W. Cold stress activates disease resistance in Arabidopsis thaliana through a salicylic acid dependent pathway. Plant Cell Environ. 2019, 42, 2645-2663. [CrossRef]

63. Chun, H.J.; Baek, D.; Cho, H.M.; Lee, S.H.; Jin, B.J.; Yun, D.J.; Hong, Y.S.; Kim, M.C. Lignin biosynthesis genes play critical roles in the adaptation of Arabidopsis plants to high-salt stress. Plant Signal. Behav. 2019, 14, 1625697. [CrossRef] [PubMed]

64. Molin, W. Glyphosate, a Unique Global Herbicide. J. E. Franz, M.K. Mao, and J. A. Sikorski, ACS Monograph 189, 1997.653 pp. Weed Technol. 1997, 12, 564-565. [CrossRef]

65. Yang, X.; Beres, Z.T.; Jin, L.; Parrish, J.T.; Zhao, W.; Mackey, D.; Snow, A.A. Effects of over-expressing a native gene encoding 5enolpyruvylshikimate-3-phosphate synthase (EPSPS) on glyphosate resistance in Arabidopsis thaliana. PLoS ONE 2017, 12, e0175820. [CrossRef] [PubMed]

66. Fang, J.; Nan, P.; Gu, Z.; Ge, X.; Feng, Y.-Q.; Lu, B.-R. Overexpressing exogenous 5-Enolpyruvylshikimate-3-Phosphate Synthase (EPSPS) genes increases fecundity and auxin content of transgenic Arabidopsis plants. Front. Plant Sci. 2018, 9, 233. [CrossRef]

67. Alscher, R.G.; Erturk, N.; Heath, L.S. Role of superoxide dismutases (SODs) in controlling oxidative stress in plants. J. Exp. Bot. 2002, 53, 1331-1341. [CrossRef] 
68. Elstner, E.F. Mechanisms of oxygen activation in different compartments of plant cells. In Active Oxygen/Oxidative Stress and Plant Metabolism; Pell, E.J., Steffen, K.L., Eds.; American Society of Plant Physiologists Press: Rockville, MD, USA, 1991; Volume 1, pp. 13-25.

69. Cai, Y.T.; Zhang, H.; Qi, Y.P.; Ye, X.; Huang, Z.-R.; Guo, J.-X.; Chen, L.-S.; Yang, L.-T. Responses of reactive oxygen species and methylglyoxal metabolisms to magnesium-deficiency differ greatly among the roots, upper and lower leaves of Citrus sinensis. BMC Plant Biol. 2019, 19, 76. [CrossRef] [PubMed]

70. Mhamdi, A.; Queval, G.; Chaouch, S.; Vanderauwera, S.; Van Breusegem, F.; Noctor, G. Catalase function in plants: A focus on Arabidopsis mutants as stress-mimic models. J. Exp. Bot. 2010, 61, 4197-4220. [CrossRef]

71. Escobar, J.A.; Rubio, M.A.; Lissi, E.A. SOD and catalase inactivation by singlet oxygen and peroxyl radicals. Free Radic. Biol. Med. 1996, 20, 285-290. [CrossRef]

72. Orendi, G.; Zimmermann, P.; Baar, C.; Zentgraf, U. Loss of stress-induced expression of catalase3 during leaf senescence in Arabidopsis thaliana is restricted to oxidative stress. Plant Sci. 2001, 161, 301-314. [CrossRef]

73. Yamamoto, Y.; Kobayashi, Y.; Matsumoto, H. Lipid Peroxidation Is an Early Symptom Triggered by Aluminum, But Not the Primary Cause of Elongation Inhibition in Pea Roots. Plant Phys. 2001, 125, 199-208. [CrossRef]

74. Hasanuzzaman, M.; Nahar, K.; Hossain, M.S.; Mahmud, J.A.; Rahman, A.; Inafuku, M.; Oku, H.; Fujita, M. Coordinated actions of glyoxalase and antioxidant defense systems in conferring abiotic stress tolerance in plants. Int. J. Mol. Sci. 2017, 18, 200. [CrossRef] [PubMed]

75. Smith, C.A.; O'Maille, G.; Want, E.J.; Qin, C.; Trauger, S.A.; Brandon, T.R.; Custodio, D.E.; Abagyan, R.; Siuzdaket, G. METLIN: A metabolite mass spectral database. Ther. Drug Monit. 2005, 27, 747-751. [CrossRef] [PubMed]

76. Xia, J.; Mandal, R.; Sinelnikov, I.; Broadhurst, D.; Wishart, D.S. MetaboAnalyst 2.0—a comprehensive server for metabolomic data analysis. Nucleic Acids Res. 2012, 40, W127-W133. [CrossRef] [PubMed]

77. Bertini, L.; Cozzolino, F.; Proietti, S.; Falconieri, G.S.; Iacobucci, I.; Salvia, R.; Falabella, P.; Monti, M.; Caruso, C. What antarctic plants can tell us about climate changes: Temperature as a driver for metabolic reprogramming. Biomolecules 2021, 11, 1094. [CrossRef] 